\title{
Einleitung: „Freiheit von Not“ als internationaler Nachkriegskonsens
}

Dass die Sicherung der grundlegendsten menschlichen Bedürfnisse wie Essen, Kleidung und Wohnung allen zustehe, ist Teil des Gründungskonsenses der internationalen Nachkriegsordnung. „Freiheit von Not" ist eine der Grundformeln der Allgemeinen Erklärung der Menschenrechte von 1948, die bereits in der Präambel formuliert wird. Ähnliche Ideen sind in den Gründungsdokumenten westlicher Wohlfahrtsstaaten in den 1940ern enthalten. $\mathrm{Zu}$ den five giants, die der welfare state zu bekämpfen habe, zählte William Beveridge in seinem nicht nur in Großbritannien einflussreichen Bericht von 1942 Want und Squalor (Beveridge 1942: 6). Das Recht auf Menschenwürde und das Sozialstaatspostulat sind Teil des westdeutschen Grundgesetzes von 1949, woraus das Bundesverwaltungsgericht 1954 einen individuellen Rechtsanspruch auf staatliche Fürsorgeleistungen im Falle von Bedürftigkeit ableitete. Armutsbekämpfung zählt zum kleinsten gemeinsamen Nenner (fast) aller westlicher Wohlfahrtsstaaten. Selbst im Strafrecht gab es noch lange die explizite Legitimation von „Mundraub“ bei akuter Bedürftigkeit.

Auch in Bezug auf nicht-westliche Entwicklungs- und Übergangsgesellschaften ist die Bekämpfung von Hunger und Armut ein zentrales Thema. Seit Ablösung der Kolonialpolitik durch „Entwicklungspolitik“ und zuletzt „Entwicklungszusammenarbeit" ist Armutsbekämpfung ein zentrales Ziel der Politik westlicher Länder und internationaler Organisationen gegenüber Ent- 
wicklungsländern. Armut ist weiterhin eine weltweite Herausforderung, auch wenn in einigen Erdteilen das Ausmaß der Armut erheblich reduziert werden konnte (Übersicht 0.1).

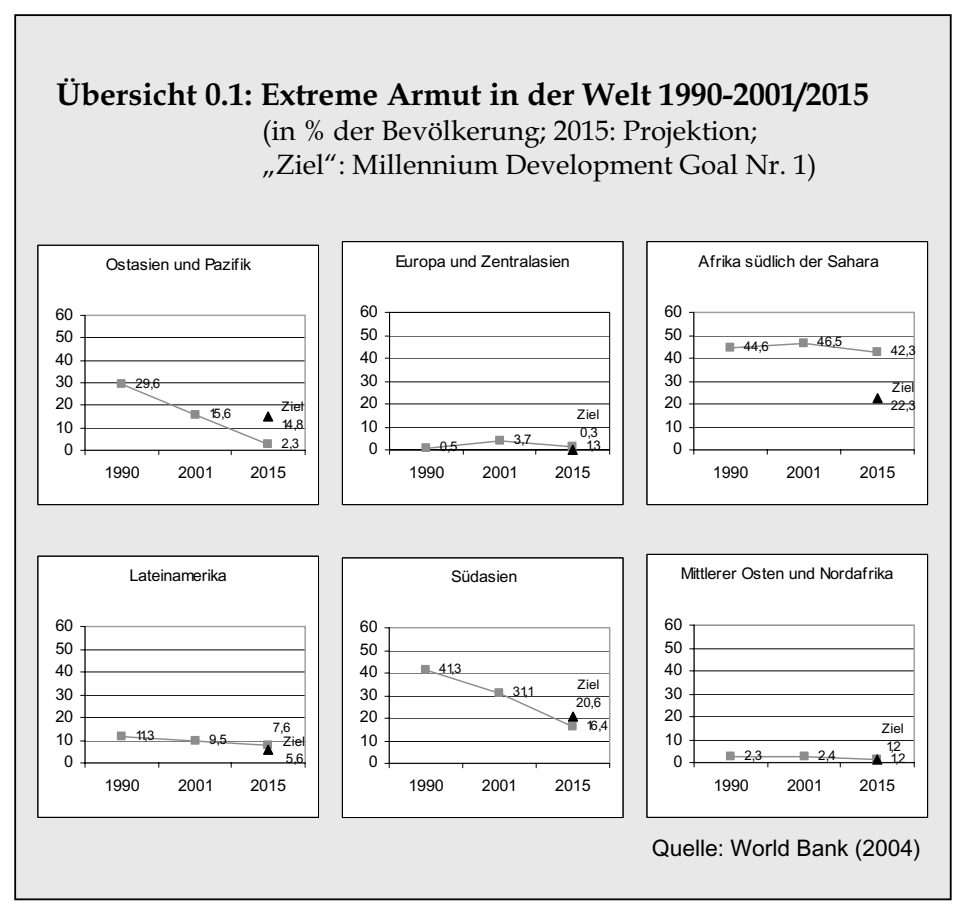

Trotz des internationalen Nachkriegskonsenses in Bezug auf, Freiheit von Not' und Bekämpfung von Armut waren soziale Grundsicherungssysteme jedoch bis vor wenigen Jahren kein globales Thema. Dies betrifft alle drei angesprochenen Politikbereiche bzw. Diskurse, in denen die Grundsicherungsidee potenziell von Bedeutung ist: In der globalen Menschenrechtspolitik und in der Entwicklungspolitik ist die Idee kaum präsent, und selbst in der Sozialpolitik der westlichen Wohlfahrtsstaaten ist sie nicht umstandslos gesichert.

Die Menschenrechtserklärungen und -konventionen berühren in vielfältiger Weise Aspekte der materiellen Lebensverhältnisse der Menschen. Die unmittelbar einschlägigen Teile, die sozialen Menschenrechte, haben jedoch einen schwächeren Status als die zivi- 
len und die politischen Menschenrechte. Zudem liegt der Schwerpunkt der Politik sozialer Menschenrechte auf arbeitsbezogenen Rechten, während das Recht auf soziale Sicherung (Artikel 9 des Internationalen Pakts über wirtschaftliche, soziale und kulturelle Rechte von 1966) ein Schattendasein führt.

In westlichen Wohlfahrtsstaaten sind Grundsicherungen fast überall eingeführt, vor allem in Form von Sozialhilfe, deren Existenz gesellschaftlich grundsätzlich unbestritten ist. Aber die Sozialhilfe ist ein ungeliebtes System - ungeliebt von Linken wie von Neoliberalen. Die Einstellungen zur Sozialhilfe sind verwickelt. So beklagen Wirtschaftsliberale oft dysfunktionale Folgen von Sozialhilfe, etwa eine Verminderung des Arbeitsanreizes, fordern zugleich aber eine Rückführung des Wohlfahrtsstaates auf Leistungen für die "wirklich Bedürftigen“, also im Kern auf eine Sozialhilfe. Zugleich gibt es in linken intellektuellen Kreisen Idealisierungen der Idee sozialer Grundsicherung zu einem allgemeinen, nicht einkommensabhängigen Grundeinkommen für alle. Die Sozialhilfe gilt dann als schlechte Grundsicherung.

In der Entwicklungspolitik dominiert das Konzept der Hilfe zur Selbsthilfe, also gerade ein Verzicht auf direkte Grundsicherungsleistungen an Arme. Hilfe zur Selbsthilfe ist Teil der übergreifenden Zielformel "Entwicklung“, verstanden als kollektive und strukturelle Verbesserung der Lebensbedingungen in Entwicklungsländern. Direkte individuenbezogene Leistungen internationaler Organisationen sind weitgehend beschränkt auf humanitäre Hilfe im Katastrophenfall und bei Flüchtlingen. Allgemeine institutionalisierte (auf Dauer gestellte) und individuelle Rechtsansprüche konstituierende Leistungssysteme fehlen. Zwischen ,Hilfe zur Selbsthilfe' und ,Entwicklung' einerseits und humanitärer Nothilfe andererseits ist eine Leerzone, ein Niemandsland. In diese Zone dringt derzeit die Idee sozialer Grundsicherung ein.

Anlass der vorliegenden Studie ist die Beobachtung, dass sich in allen drei Bereichen - Menschenrechtsschutz, Sozialpolitik, Entwicklungspolitik - seit den 1990er Jahren eine Bedeutungszunahme sozialer Grundsicherung andeutet.

Im Bereich der Menschenrechte ist zum einen feststellbar, dass der Menschenrechtsdiskurs zunehmend in die Politik der Armutsbekämpfung hineingetragen, Armut also verstärkt als Menschenrechtsproblem definiert wird. Zum anderen erfährt das Recht auf soziale Sicherheit aus dem Pakt von 1966 im zuständi- 
gen Menschenrechtsausschuss der Vereinten Nationen seit 2006 erstmals nähere Aufmerksamkeit.

Im Bereich der Sozialpolitik in westlichen Ländern haben sozialhilfeartige Leistungen an Bedeutung gewonnen, weil Erwerbs-, Familien- und Migrationsbiographien wieder riskanter und diskontinuierlicher geworden sind. ${ }^{1}$ Die Sozialhilfe ist von einem System am Rande der Sozialpolitik zu einem zentralen Thema der gesellschaftspolitischen Diskussion geworden. Zugleich ist die Sozialhilfe in den westlichen Ländern seit den 1990er Jahren in Bezug auf Ziele, Instrumente und Verwaltungsstrukturen wesentlich umgestaltet worden. Hierdurch wurde die Akzeptanz der Sozialhilfe in Wirtschaftskreisen gesteigert und der Typus „moderne Sozialhilfe", nach dessen Export in nicht-westliche Länder wir fragen, modernisiert. Die Sozialhilfe bzw. (als Genotyp) die Fürsorge verschwindet nicht, sondern wird in Abständen von mehreren Jahrzehnten immer wieder reformiert (in Deutschland 1924, 1961 und 2003/2005).

Im Bereich der Entwicklungspolitik schließlich gibt es von Seiten einiger internationaler Organisationen wie von Regierungen mehrerer Entwicklungs- und Übergangsgesellschaften Ansätze zur Etablierung sozialer Grundsicherungssysteme. Ein Anlass ist das Auftreten eines neuen Inklusionsproblems: Auf bestimmte neue oder verstärkt wahrgenommene Problemgruppen - vor allem AIDS-Familien, alleinerziehende Frauen, alte Menschen und Menschen mit Behinderungen - ist das Konzept der Hilfe zur Selbsthilfe nicht ohne weiteres anwendbar, da sie nur begrenzt selbsthilfefähig sind. Für diese Gruppen werden soziale Grundsicherungen erprobt.

Es ist eine offene Frage, ob sich die drei grundsicherungsrelevanten Diskursstränge bzw. Politikbereiche (Menschenrechte, Entwicklungspolitik, Sozialpolitik) berühren werden. Wenn eine Verknüpfung gelänge, könnte es Synergieeffekte geben im Hinblick auf eine verstärkte Verbreitung von Grundsicherungssystemen. Die vorliegende Studie ist selbst Ausdruck einer Interaktion zwischen Entwicklungspolitik und Sozialpolitik(forschung). Denn in der Studie werden Konzepte und Methoden der westlichen So-

1 Zu Sozialhilfe als biographische Kontinuität herstellende Form sozialer Risikobearbeitung s. Leisering (2003b: 212f.). 
zialpolitikforschung erstmals auf Fragen sozialer Grundsicherung in Entwicklungsländern angewendet.

Auslöser der Studie war eine Interaktion von Politik und Wissenschaft: Die deutsche Gesellschaft für Technische Zusammenarbeit (GTZ) führt Modellprojekte zu Grundsicherung in Afrika durch. Ihr Auftraggeber, das Bundesministerium für wirtschaftliche Zusammenarbeit und Entwicklung (BMZ), hat zur Exploration der neuen Strategie eine Studie angestoßen, auf der das vorliegende Buch in seinem empirischen Teil basiert (Leisering/Buhr/ Traiser-Diop 2004, GTZ 2006). Pionier der Idee einer sozialen Grundsicherung für Entwicklungsländer war in Deutschland Bernd Schubert, der seit den 1980er Jahren diese - wahlweise als abwegig, revolutionär oder im heutigen Kontext der Globalisierung von Sozialpolitik als überfällig zu sehende - Idee gegen viele Vorbehalte entwickelt hat. Schubert veröffentlichte 1985 einen Artikel „Eine Rente für die Ärmsten“ in Die Zeit (s. Seminar für ländliche Entwicklung 2003: 11). Parallel zur vorliegenden Studie hat Schubert einen Bericht zur Rolle sozialer Grundsicherung in den Konzepten der Organisationen internationaler Zusammenarbeit erstellt (Schubert 2004; Auszüge als Kapitel 17 des vorliegenden Buches). In der internationalen Zusammenarbeit macht sich vor allem das britische Department for International Development (DFID) seit einigen Jahren für soziale Grundsicherungen in Entwicklungsländern stark.

\section{Ziele, Aufbau und Fragestellungen der Studie}

Ziel der Studie ist, Grundsicherungssysteme in westlichen und nicht-westlichen Ländern zu untersuchen, um ihre Verbreitung, ihre Formen, ihre Wirkungen und ihre gesellschaftlichen Voraussetzungen zu identifizieren. Dabei betritt die Studie in mehrfacher Hinsicht Neuland.

Neu ist erstens die in diesem Buch konzeptuell und empirisch vorzustellende Strategie, Armut in Entwicklungs- und Übergangsgesellschaften auch durch sozialhilfe- oder rentenartige Leistungen (Grundsicherungen) zu bekämpfen. Denn herkömmlich versteht sich Entwicklungspolitik als Hilfe zur Selbsthilfe. Bloße hand outs wie Sozialhilfe gelten als nicht nachhaltig, ja als kontraproduktiv für die Hilfeempfänger wie für die gesellschaftliche Entwicklung. Auch die Sozialhilfe in westlichen Ländern sieht sich, besonders von liberalökonomischer Seite, einer derartigen Kritik 
ausgesetzt. Grundsicherungen in Entwicklungs- und Übergangsgesellschaften sind bislang primär in Berichten aus der Entwicklungszusammenarbeit, nur wenig und selektiv in der wissenschaftlichen Literatur behandelt worden.

Neu ist zweitens, dass hier erstmals ein weltweiter Survey über soziale Grundsicherungen vorgelegt wird, verbunden mit einer Definition des schwer fassbaren und vielgestaltigen Begriffs "Grundsicherung". Es ist der erste umfassende und systematische Überblick über Grundsicherungen in Entwicklungs- und Übergangsgesellschaften (und in entwickelten Ländern, s.u.). ${ }^{2}$ Bislang liegen für Entwicklungs- und Übergangsländer nur Studien zu einzelnen Systemtypen, z.B. zu beitragsfreien Rentensystemen, und zu einzelnen Ländern oder Weltregionen vor. Durch die neue Gesamtsicht werden verschiedene Typen sozialer Sicherung, die bisher in getrennten Literatursträngen behandelt wurden, erstmals als Varianten sozialer Grundsicherung identifiziert und in einen einheitlichen Bezugsrahmen gestellt. Wir schlagen vor, fünf solche Typen von Grundsicherung zu unterscheiden. Zudem wird durch den weltweiten Survey erstmals erkennbar, dass sich soziale Grundsicherungen bereits seit den 1990er Jahren weit verbreitet haben. Die ideologischen Kämpfe für und wider Grundsicherungssysteme sind insoweit durch die Wirklichkeit bereits überholt.

Drittens ist die vorliegende Studie u.W. der erste systematische Versuch, Sozialhilfe und Grundsicherung in nicht-westlichen Ländern mit den Mitteln der an westlichen Ländern geschulten Forschung zu untersuchen und damit in das neue Forschungsgebiet global social policy einzuführen. Die Autoren können hierbei auf ihre umfangreichen Erfahrungen in der Erforschung westlicher Sozialhilfesysteme zurückgreifen. Seit den 1990er Jahren beginnen westliche Sozialpolitikforscher generell, sich auch für Entwicklungs- und Übergangsgesellschaften $\mathrm{zu}$ interessieren und die Methoden und Theorien, die für westliche Wohlfahrtsstaaten entwickelt wurden, in modifizierter Form auf nicht-westliche

2 Die Studie wurde zuerst als Arbeitspapier vorgelegt (Leisering/ Buhr/Traiser-Diop 2004). Daneben gibt u.W. nur DFID (2005) eine systematische Gesamtdarstellung des Konzepts sozialer Grundsicherung, allerdings nur mit selektiven Länderbeispielen (partielle, anders geartete Überblicke gaben z.B. Howell 2001 a,b und Tabor 2002). 
Länder anzuwenden. Die globale Ausdehnung der westlichen Sozialpolitikforschung reagiert auf die reale Globalisierung der Sozialpolitik: die Verbreitung westlicher Modelle und die Entstehung von global social policy (Deacon 1987). Global Social Policy ist zugleich der Name des neuen Forschungsparadigmas (s. die neue Zeitschrift Global Social Policy, 2001 ff.). Die Kernbereiche sozialer Sicherung, Alterssicherung und Gesundheit, sind bereits etablierte Themen des globalen sozialpolitischen Diskurses und der global social policy-Forschung, nicht jedoch die Sozialhilfe. Unsere Studie zeigt, dass auch der weniger aufmerksamkeitsträchtige Bereich monetärer Mindestsicherung zu einer Frage globaler Sozialpolitik geworden ist und entsprechende Beachtung verdient. Der Bedeutungszuwachs von global social policy geht einher mit einer Veränderung eingeführter Politikfelder wie auch Forschungsgebiete: Entwicklungspolitik und Sozialpolitik berühren und überlappen sich seit den 1990ern zunehmend. In den Gebieten Alter und Gesundheit ist dies bereits greifbar, im Bereich soziale Grundsicherung noch nicht. Die vorliegende Arbeit versteht sich daher auch als Beitrag, die Entwicklungspolitik durch Konzepte und Perspektiven der westlichen Sozialpolitik und Sozialpolitikforschung zu befruchten.

Viertens ist es der erste Versuch, Institutionen und Probleme der Grundsicherung und Sozialhilfe in entwickelten und Entwicklungsländern empirisch und theoretisch systematisch zusammen $z u$ behandeln und Anstöße für eine übergreifende Theoriebildung zu geben. Hierzu wird ein bislang nicht verfügbarer Überblick über westliche Systeme auf dem neuesten Stand der Forschung gegeben und auch danach gefragt, wovon nicht-westliche Länder lernen können und wovon nicht. Insgesamt wird also ein wirklich weltweiter Überblick gegeben. Chancen und Probleme von Grundsicherungen sind in entwickelten und Entwicklungsländern teilweise vergleichbar. Auch die Diskurse sind erstaunlich ähnlich. Sozialhilfeartige Sicherungssysteme sind in beiden Kontexten vielfach ungeliebt, grundsätzliche Vorbehalte kommen insbesondere von liberalökonomischer Seite. Die Zusammensicht westlicher und nicht-westlicher sozialer Sicherungssysteme soll auch exemplarisch dazu beitragen, das neue Forschungsparadigma global social policy im vollen Sinne, global' zu machen. Denn, global social policy' bezieht sich bislang primär auf die Aktivität internationaler Organisationen und auf Sozialpolitik in Entwicklungs- und 
Übergangsgesellschaften, ohne systematischen Einbezug westlicher nationaler Sozialpolitik. Eine Zusammensicht westlicher und nicht-westlicher Systeme findet sich in der Literatur i.a. nur auf der Ebene ganzer Wohlfahrtsregime, etwa als Frage, ob das japanische oder das chinesische Wohlfahrtssystem einem der EspingAndersenschen "Wohlfahrtsregime“" nahe kommt (und wenn ja, welchem) oder ob es eigene ostasiatische Wohlfahrtsregime gibt (Esping-Andersen 1997, Gough/Wood 2004).

Die Studie ist in fünf Teile gegliedert. Im ersten Teil "Soziale Grundsicherung als globale Herausforderung" (Kapitel 1), wird das Bezugsproblem der Untersuchung präzisiert: Inwieweit ist die Frage sozialer Grundsicherung eine globale Herausforderung? Zudem wird die Rolle der Grundsicherungsidee in Entwicklungsund Sozialpolitik näher bestimmt und der Begriff "Grundsicherung" analytisch präzisiert. Der zweite Teil (Kapitel 2-5) gilt der Hauptform sozialer Grundsicherung in entwickelten Gesellschaften, der Sozialhilfe (social assistance, welfare). Hier wird ein bisher nicht verfügbarer umfassender, aber konzentrierter Überblick auf dem neuesten Stand der (in den letzten Jahren angewachsenen) Forschung gegeben. Der dritte Teil (Kapitel 6-12) thematisiert das breite Spektrum sozialer Grundsicherungssysteme in Entwicklungs- und Übergangsgesellschaften. Dieses Spektrum wird auf fünf Haupttypen reduziert. Aufbauend auf den Analysen im zweiten und dritten Teil werden im vierten Teil entwicklungspolitische Strategien in Bezug auf die Einführung sozialer Grundsicherungssysteme in Entwicklungs- und Übergangsgesellschaften analysiert: Voraussetzungen und Rahmenbedingungen von Grundsicherung, institutionelle Optionen, Gestaltungs- und Wirkungsprobleme und die Rolle von Grundsicherung in den Strategien internationaler Organisationen (Kapitel 13-17). Es folgen ein Fazit und weltgesellschaftstheoretische Erklärungsansätze zur Entwicklung sozialer Grundsicherung (fünfter Teil, Kapitel 18-20).

Die Studie basiert auf einer Analyse der vorliegenden Literatur und eigenen Forschungen zur deutschen, britischen, schwedischen, chinesischen und südkoreanischen Sozialhilfe (Leisering 2006, Leisering/Leibfried 1999, Berner/Leisering 2003, Leisering/Hilkert 2000, Buhr 1995, 1999a, 2003, Leisering/Gong/ Hussain 2002; Kim 2006).

Im einzelnen geht die Untersuchung folgenden Fragen nach: 
1. In welchen globalen Zusammenhängen stellen sich in jüngerer Zeit Fragen sozialer Grundsicherung? (Abschnitt 1.1) Warum wurde das Thema lange ignoriert und ist noch heute vielfach prekär? (Abschnitte 1.1-1.3)

2. Was beinhaltet der Begriff "soziale Grundsicherung", welche Dimensionen und Varianten gibt es? (Abschnitt 1.4) Welches sind die Konzepte sozialer Grundsicherung in der Entwicklungspolitik und in der Sozialpolitik westlicher Wohlfahrtsstaaten? (Abschnitte 1.2, 1.3 und Kapitel 6)

3. Was sind die Ziele, institutionellen Formen, Wirkungen und Probleme sozialer Grundsicherung (primär Sozialhilfe) in westlichen Wohlfahrtsstaaten? (Gemeinsamkeiten, Unterschiede; Kapitel 3-5) Welche Rolle spielt die Sozialhilfe in unterschiedlichen Wohlfahrtsstaaten? („Sozialhilferegime“; Kapitel 2) Gibt es einen Idealtypus westlicher "moderner Sozialhilfe“? (Abschnitt 3.8) Inwieweit ist dieser Typus auf nicht-westliche Länder transferierbar? (Kapitel 13, 15)

4. Wie verbreitet sind soziale Grundsicherungssysteme in Entwicklungs- und Übergangsgesellschaften? (Kapitel 7-11)

5. Welche Arten von Grundsicherungssystemen gibt es in diesen Ländern? (Kapitel 6-12)

6. Wie wirksam sind diese Grundsicherungssysteme? Haben sie Folgen über die unmittelbar intendierten Wirkungen hinaus? (Kapitel 6-12)

7. Welcher gesellschaftlichen Voraussetzungen bedarf es in Entwicklungs- und Übergangsgesellschaften, um Grundsicherungen zu institutionalisieren? (Kapitel 14)

8. Inwieweit haben Grundsicherungen in die Programmatik internationaler Organisationen und nationaler Entwicklungsorganisationen (wie die GTZ) Eingang gefunden? (Kapitel 17)

9. Sind soziale Grundsicherungen alles in allem sinnvolle Instrumente im Repertoire globaler Entwicklungs- und Sozialpolitik? Was ist ihr Ort in Entwicklungsstrategien? (Kapitel 18) Welchen Ort hat die Idee sozialer Grundsicherung in globalen sozialen Diskursen? (Kapitel 19) Welches sind die politischen Aussichten weiterer globaler Verbreitung des neuen Instruments? Ist Grundsicherung Teil der Weltgesellschaft? (Kapitel 20). 
